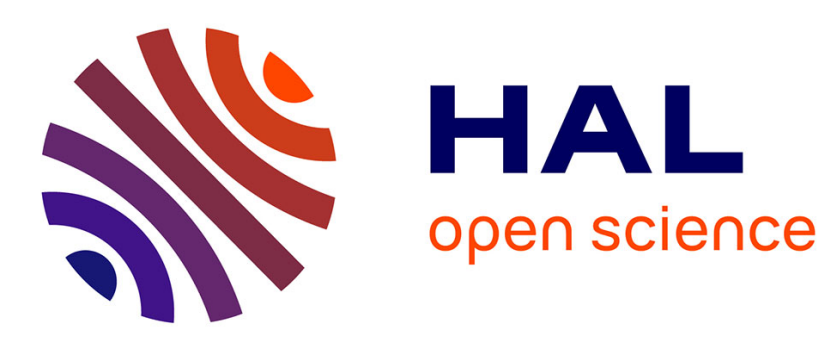

\title{
A nonlinear numerical simulation of a lab centrifuge with internal damping
}

Jonas Fischer, Jens Strackeljan

\section{To cite this version:}

Jonas Fischer, Jens Strackeljan. A nonlinear numerical simulation of a lab centrifuge with internal damping. Nonlinear Dynamics, 2009, 60 (1-2), pp.39-47. 10.1007/s11071-009-9578-9 . hal-00568393

\section{HAL Id: hal-00568393 \\ https://hal.science/hal-00568393}

Submitted on 23 Feb 2011

HAL is a multi-disciplinary open access archive for the deposit and dissemination of scientific research documents, whether they are published or not. The documents may come from teaching and research institutions in France or abroad, or from public or private research centers.
L'archive ouverte pluridisciplinaire HAL, est destinée au dépôt et à la diffusion de documents scientifiques de niveau recherche, publiés ou non, émanant des établissements d'enseignement et de recherche français ou étrangers, des laboratoires publics ou privés. 


\title{
A nonlinear numerical simulation of a lab centrifuge with internal damping
}

\author{
Jonas Fischer and Jens Strackeljan
}

Received: date / Accepted: date

\begin{abstract}
This paper is about numerical simulations of dissipation processes in rotor shaft joints of rotor systems. Based on measurement results a nonlinear simulation model of a lab centrifuge is stated. The effects of internal damping in combination with nonlinear stiffness and friction in the rotor shaft joint of the lab centrifuge are worked out. It is shown that the nonlinearities cause the amplitudes to rest limited once increased amplitudes due to internal damping appear. One focus is the derivation of suitable force laws describing the mechanisms of the components within the connection.
\end{abstract}

Keywords rotordynamics · internal damping . nonlinearity $\cdot$ rotor shaft joint

\section{Introduction}

Particularly in the field of lab centrifuges problems like rough running, high excitations of the rotor or even rub between housing and rotor can occur in the post critical speed range. The problems are sometimes traced back to internal damping. The word internal means, that damping and friction act in the rotating part of the rotor system. Internal damping influences the stability of the rotor system in the post critical speed range significantly.

Often the joint between rotor and shaft is the main cause for internal damping and internal friction. The mechanisms of energy dissipation within the connection are in many cases nonlinear making a simulation

Jonas Fischer and Jens Strackeljan

Institute of Applied Mechanics, Otto-von-Guericke University, Magdeburg, Germany

E-mail: jonas.fischer@ovgu.de demanding. Nevertheless a simulation remains worthwhile as it assists to analyze the reasons of internal damping and to find measures to enlarge the stability margins.

In the present article a lab centrifuge is examined where high rotor deflections due to internal friction are observed above a certain speed of revolution. Thereby the occurrence of high rotor deflections and rough running depends decisively on the height of the systems excitation by an impact.

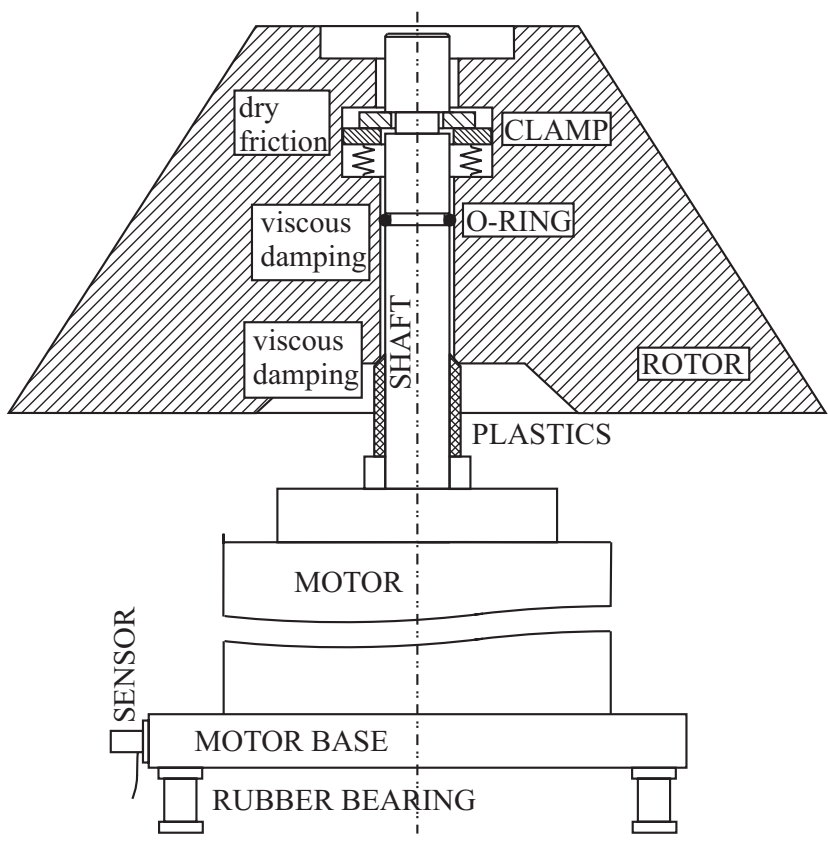

Fig. 1 Schematic model of the lab centrifuge (without safety containement and housing). 
A sketch of the lab centrifuge is shown in figure 1 . The rotor shaft joint is designed tool free with a plug and lock mechanism. It enables an easy change of rotors as this single centrifuge runs with several different rotors.

The connection between rotor and shaft consists of three parts: a clamp used to lock the connection, an o-ring which is used to center the rotor and a plastics. The plastics serves as guidance for the rotor.

In figure 2 and 3 the accelerations which were measured at the motor base of the lab centrifuge are shown. The unit was operating at a frequency of revolution of $125 \mathrm{~Hz}$. An additional excitation was performed with an impact hammer hitting the motor base. Depending in the height of the excitation increased amplitudes or a damped oscillation could be observed. The increased oscillation is bounded.

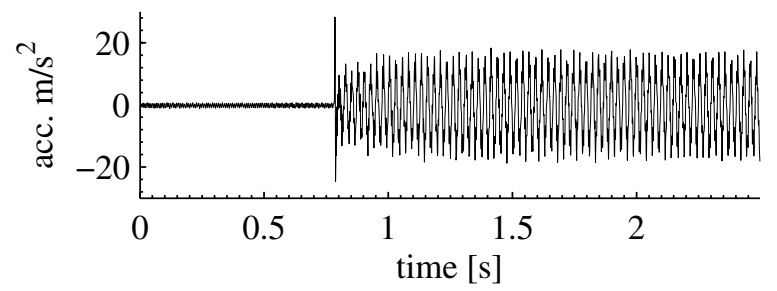

Fig. 2 Measured accelerations at the motor base after a high excitation.

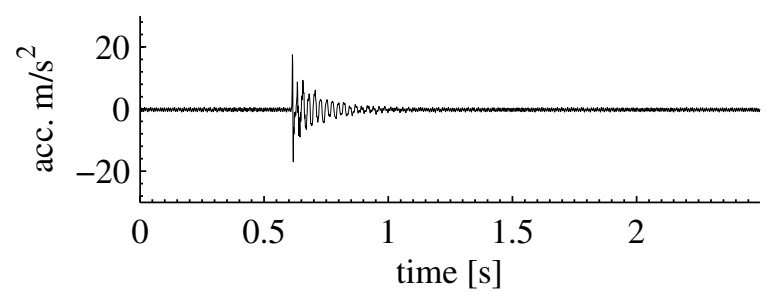

Fig. 3 Measured accelerations at the motor base after a low excitation.

One can assume that the clamp opens if the force in the connection clamp is higher than the friction force. This is the case, if the impact at the motor base is high. Thus the internal damping of the o-ring starts to work. From literature it is known, that linear internal damping causes the amplitudes to rise unbounded ([1]). As the amplitudes are bounded in figure 2, the force laws for o-ring and plastics have to be nonlinear. The stiffness of o-ring and plastics are nonlinear in this case due to the clearance between rotor and shaft.

A nonlinear simulation was performed to analyze the mechanisms of the described phenomenon exactly and to identify the main parameters influencing the stability of this system. The results are depicted in section 4.1 .

In section 2 the simulation model of the lab centrifuge and the nonlinear model of the rotor shaft joint is explained. A linear eigenvalue simulation is used to evaluate the simulation parameters. The results can be found in section 3 .

\section{Simulation model}

In figure 4 one can see a sketch of the simulation model. It consists of an elastic shaft, a rigid rotor and three linear spring-damper-elements describing the ball bearings between stator and shaft and the rubber bearing. The connection between rotor and shaft is modelled by three nonlinear elements.

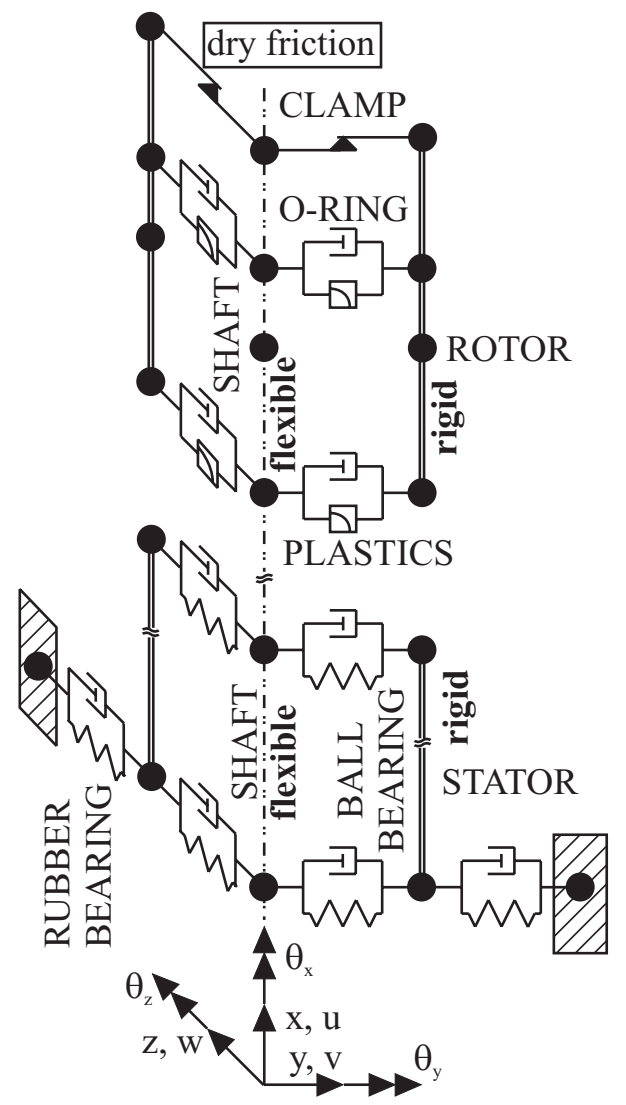

Fig. 4 Simulation model of the lab centrifuge. Coordinate system $x, y, z$ with displacements $u, v, w$ (translation) and $\theta_{x}, \theta_{y}, \theta_{z}$, (rotation). 
The dynamic behaviour of the rotor system is described by the discrete differential equation in the inertial coordinate system.

$$
[M]\{\ddot{D}\}+[[C]+[G]]\{\dot{D}\}+[K]\{D\}=\left\{F^{e x t}\right\}+\left\{F^{n l}\right\}
$$

with stiffness matrix $[K]$, damping matrix $[C]$, gyroscopic matrix $[G]$ and mass matrix $[M]$. Vector $\{D\}$ consists of the nodal displacements, vector $\left\{F^{\text {ext }}\right\}$ of the linear external and $\left\{F^{n l}\right\}$ of the non-linear internal and external forces.

To obtain equation (1), the principle of linear momentum is first stated in a body fixed coordinate system and subsequently transformed into the inertial coordinate system.

The internal forces $\left\{F^{\text {int }}\right\}^{*}=[K]^{*} \cdot\{D\}^{*}+[C]^{*} \cdot\{\dot{D}\}^{*}$ describing the stiffness and damping properties of the shaft and the linear part of the shaft rotor connection in a coordinate system rotating with the frequency of revolution are transformed into the inertial coordinate system. The ${ }^{*}$ denotes that the entity is used in the rotating coordinate system.

The shaft of the rotor system is discretised by 8 beam elements, including transverse shear deformation, according to Timoshenkos beam theory ([2]). The upper and lower shaft bearing of the centrifuge and the nodes of the rotor are coupled rigidly. Constraints are used to connect the movements of the corresponding nodes.

The parameters to state the stiffness and mass matrix of the shaft follow directly from the shafts geometry and material properties. Linear stiffness and damping coefficients of the rubber support are deduced by separate tests. The inertia moments of the rotor are obtained numerically.

\subsection{Simulation model of the rotor shaft joint}

On the one hand the mechanisms of damping which occur in the rotor shaft joint can be traced back to material damping. This is for example the case for the o-ring connecting rotor and shaft. Another mechanism are micromovements and as a result friction between the single components of the rotor shaft connection.

The energy dissipation of material damping is describable by viscous internal damping. The Voigt-Model can be used for simulation. In [3] a linear viscous internal damping force is deduced from this material model. The force law is extended by a nonlinear part if huge amplitudes are considered. In the linear case, one can expect unbounded amplitudes above a certain frequency of revolution. In [4] a similar nonlinear force law is applied. It is shown that the analytical solution of the considered rotor system is bounded for all frequencies.
A fundamental analyze of rotating dry friction can be found in [5]. The analytical steady state solution and an investigation about the stability of the solution is accomplished by measurement results.

Newer works principally deal with the description of micromovements and the resulting structural damping in practical applications.

There exists different approaches to model friction between the single components of a connection.

By one hand measured hysteresis are described by phenomenological models, like for example the DahlModel [6]. This approach does not allow to predict directly the motion of the rotor system. Parameters are determined for each component of a device, for which hysteresis loops were measured. The parameters are subsequently used for a description of the whole device.

On the other hand there exist micro slip models like for example the model of Masing or of Menq [7] or models, which take into account the tip elasticity of the coarsens of the contact area like in [8] and [9]. Micro slip means that in the beginning only one part of the contact area is slipping prior the entire contact area slips.

In rotor dynamics micro slip models are used since some years especially for modelling the processes between turbine blades and shaft respectively the processes in spline shafts. In [10] the dissipation energy caused by micro slip and material damping in slot wedges in a turbine generator are analyzed.

Furthermore it is possible to simulate the connection with contact elements of the finite element method instead of the use of micro slip models. In [11] a technique is proposed to calculate the nonlinear response of an harmonic excited turbomachine at constant speed of revolution. Blades and rotorshaft are generated as finite element model. Contact elements are used for the joint. The simulation is done by the DLFT-method (Dynamical Lagrange Frequency Time Method, [12]), assuming that only periodic forces and deflections occur. Measurements show a good agreement with the simulation results. But there is no possibility to take into account arbitrary excitations, like impact forces or to compute a run-up of the rotor system.

As simulation approach for the considered lab centrifuge, the rotor shaft joint is divided in parts which are different in construction. For every part of the joint a nonlinear force law is stated. Parameters which are necessary to describe the mechanisms of every component of the connection are determined in separate tests. This is done for example by recording hysteresis in a hydro-puls test rig. Thereby one obtains a connection model which is designed comparatively easy and which 
is suitable for transient calculations. The model is sufficiently exact to determine the influence of the single mechanisms.

The rotor shaft connection is modelled with three nonlinear elements. The choice of parameters and of force laws is explained in the following.

\subsubsection{Nonlinear force law clamp}

If the force in the connection is higher than the friction force, the clamp opens and dry friction occurs.

The rigid link between shaft node and rotor node can be implemented in the simulation model in different ways. The nodes can be linked by lagrange multipliers or by penalty-functions.

If lagrange multipliers are used, an implicit time integration method has to be used for solving the equation of motion. As the model is nonlinear, a Newton iteration in each time step is necessary to obtain the nonlinear forces.

The penalty method enables the use of an explicit integration method or a linear implicit direct integration method. But it is sometimes difficult to find appropriate penalty values.

For the simulation of the clamp a third way was chosen:

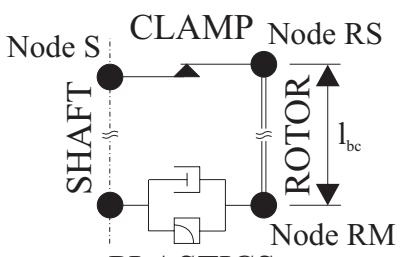

PLASTICS

Fig. 5 Constraint model clamp.

if the clamp is closed the motion of shaft node (node $\mathrm{S}$ in figure 5) and rotor node (node $\mathrm{RS}$ ) is coupled directly by constraints. Node RS is a slave node which is connected rigidly with the rotor master node RM in a distance of $l_{b c}$. Hence the equation coupling the motion of the rotor master node and the shaft node $\mathrm{S}$ by the constraint matrix $\left[\tilde{t}_{c}\right]$ is:

$\underbrace{\left\{\begin{array}{c}v_{S} \\ w_{S}\end{array}\right\}}_{\left\{d_{S}\right\}}=\underbrace{\left[\begin{array}{ccccc}1 & 0 & \cdots & 0 & l_{b c} \\ 0 & 1 & \cdots & -l_{b c} & 0\end{array}\right]}_{\left[\tilde{t}_{c}\right]} \underbrace{\left\{\begin{array}{c}v_{R M} \\ w_{R M} \\ \vdots \\ \theta_{y R M} \\ \theta_{z R M}\end{array}\right\}}_{\left\{d_{R M}\right\}}$

The entities with index $S$ are the deflections of the shaft node and the ones with index $R M$ are the deflection of the rotor master node. If we consider the reduced deflection vector $\left\{D_{r}\right\}$ of the whole system, containing only the master node deflections and the deflection vector $\{D\}$ of the non coupled system, we get the constraint matrix $\left[T_{c}\right]$.

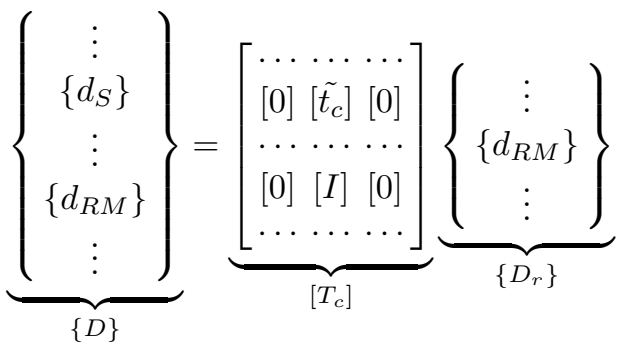

The number of degrees of freedom of the whole system is reduced by two if the clamp is closed. The equation of motion is transformed completely by the transformation matrix $\left[T_{c}\right]$. As the clamp opens the amount of degrees of freedom changes.

After each time step of the numerical integration, the state of the clamp is checked. If the force in the closed connection $F_{c c}^{*}$ is higher than the maximum adhesive force $F_{s}$, the clamp opens and a nonlinear dry friction force $F_{c}^{*}$ acts between both nodes (equation 5 ). The sticking condition is

$\left|F_{c c}^{*}\right|<F_{s}=\mu_{0} \cdot F_{N}^{*}$

In the simulation a friction coefficient $\mu_{0}=0.8$ is used, according to the combination steel - steel ([13]). The normal force $F_{N}^{*}$ is obtained from the preliminary tension force of the spring element. Equation (5) describes the dry friction force $F_{c}^{*}$ in the rotating coordinate system.

$F_{c}^{*}=-\mu \cdot F_{N}^{*} \cdot \frac{\Delta \dot{D}^{*}}{\left|\Delta \dot{D}^{*}\right|}$

The force $F_{c}^{*}$ is transformed into the inertial coordinate system. The friction coefficient in sliding state $\mu$ is assumed to be $5 \%$ smaller than $\mu_{0}$ in the sticking condition. The variable $\Delta \dot{D}^{*}$ denotes the relative velocity between rotor node and shaft node, expressed in the rotating coordinate system.

\subsubsection{Nonlinear force law o-ring}

In order to find a force law, which describes the properties of the o-ring the test configuration shown in figure 6 was build. The test configuration with two o-rings was analyzed in a hydro pulse unit. The rotor was charged with a harmonic force. The frequency was $1.0 \mathrm{~Hz}$ and the amplitude of the force was $23.75 \mathrm{~N}$. The clearance between the shaft and the rotor was the same clearance like in the rotor shaft connection of the lab centrifuge. A 


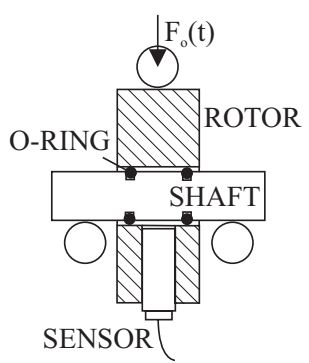

Fig. 6 Test configuration with two o-rings analyzed in a hydro pulse unit.

capacitive sensor was used to measure the displacement between rotor and shaft. The displacement is plotted in figure 7 .

The measured hysteresis loop flattens for high displacements as the clearance between rotor and shaft is reached. In addition a simulation of the test was performed nu-

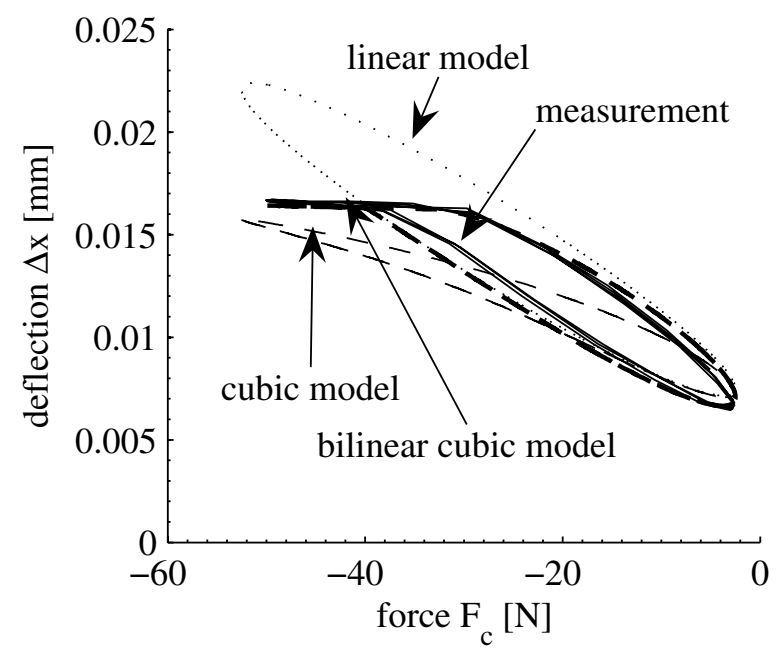

Fig. 7 Hysteresis loop of the test configuration with two o-rings, simulation and measurement.

merically. The simulation enables to deduce appropriate values for the parameters of different force laws.

The use of a linear force law for the o-ring (equation (6)) leads to an unacceptable difference between measurement and simulation.

$F_{o}=c_{i} \cdot \Delta \dot{x}+k_{i} \cdot \Delta x$

The linear damping coefficient of the o-ring is $c_{i}, k_{i}$ is the stiffness of the o-ring and $\Delta x$ is the relative displacement between shaft and rotor.

A cubic o-ring model reflects the stiffening of the oring at higher forces (equation (7)). The force deflection function remains smooth such that the numerical implementation does not provoke any difficulties. A cubic stiffness $k_{i n l}$ is used.

$F_{o}=c_{i} \cdot \Delta \dot{x}+k_{i} \cdot \Delta x+k_{i n l} \cdot \Delta x^{3}$

However this model has some drawbacks. The linear stiffness is to high and the bending of the curve is not reflected very well.

For a numerical simulation of the rotor system, a bilinear cubic force law can be used (equation (8)).

$F_{o}=c_{i} \cdot \Delta \dot{x}+k_{i} \cdot \Delta x+k_{i r} \cdot(\Delta x-s)+k_{i n l} \cdot \Delta x^{3}$

A new linear relative stiffness $k_{i r}$ is introduced which is multiplied by the difference between relative displacement $\Delta x$ and clearance $s$ and which only acts, if the relative displacement is higher than the clearance. The linear relative stiffness is much higher than the linear stiffness. The simulated and measured hysteresis loop coincide well.

\subsubsection{Nonlinear force law plastics}

The plastics is modelled similar to the o-ring, but with lower internal damping $c_{i}$ and a higher stiffness $k_{i}$ and $k_{i n l}$. As the stiffness of the plastics is much higher than that of the o-ring, it is not necessary to use a relative stiffness in the simulation. Hence the force law of equation (7) is applied.

\section{Linear simulation of the lab centrifuge}

The simulation model consists of many different parameters like stiffness properties, mass properties and damping properties. It is expensive or sometimes impossible to determine all parameters exactly. To evaluate the used parameters and to adjust them to measurement results, a linear simulation of eigenvalues and damping values was performed.

The eigenvalues were measured with a closed clamp. If the clamp of the rotor shaft joint is closed one can assume that the system is linear. Hence for the simulation of eigenvalues all nonlinear effects of the rotor shaft joint were neglected and the clamp was closed. In figure 8 the measured and calculated eigenvalues are shown.

In the linear case the damping value of the second forward whirl is important for the stability of the system. If it becomes negative the amplitude of the second forward whirl rise unbounded. The damping values corresponding to the second forward whirl of figure 8 are depicted in figure 9 . If the clamp is closed, both the measured and the simulated damping are positive up to a speed of revolution of $180 \mathrm{~Hz}$. Hence, to obtain rising 


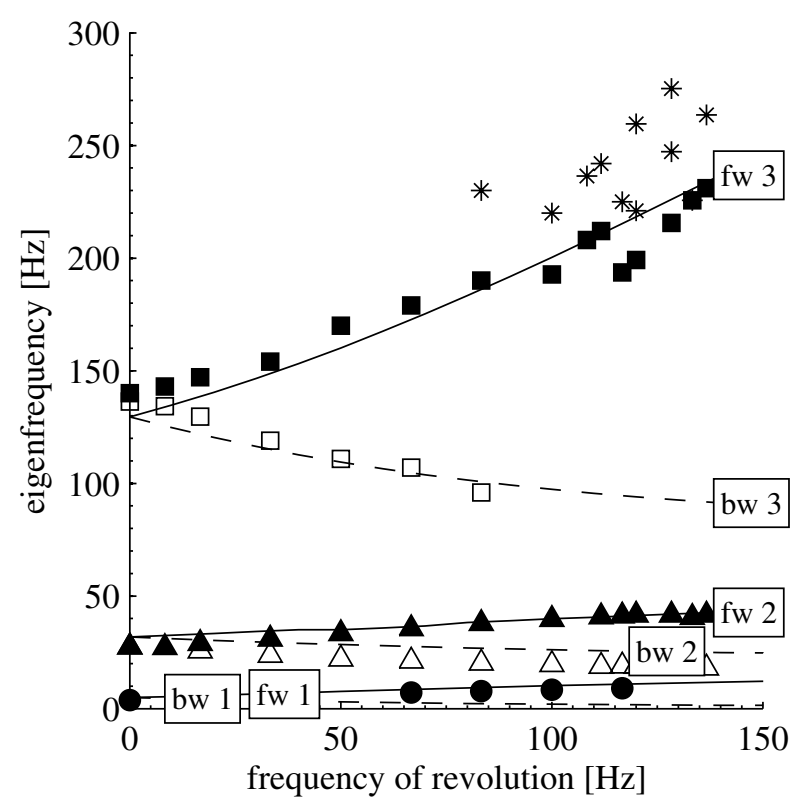

Fig. 8 Forward whirls (fw) and backward whirls (bw) of the linear simulation model (solid and dashed lines) compared to measurements (tokens).

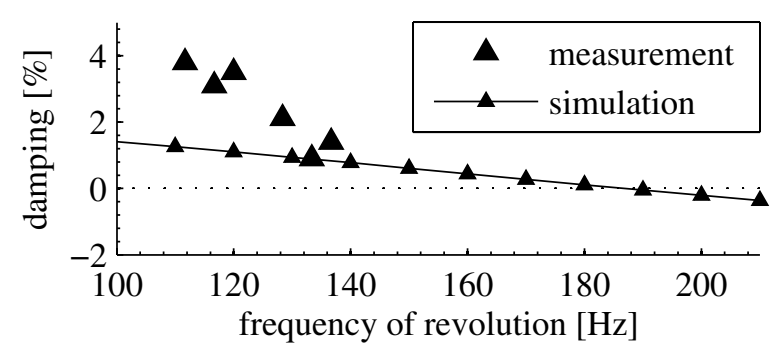

Fig. 9 Damping values of the second forward whirl. Linear simulation model compared to measurements.

amplitudes below $180 \mathrm{~Hz}$, one has to consider another mechanism, the opening of the clamp. It is not possible to describe the effect shown in figure 2 with a linear simulation model.

\section{Nonlinear simulation of the lab centrifuge}

To calculate the release of the clamp the model used for the calculation of eigenvalues was enlarged with the nonlinear properties of the rotor shaft connection. A modified Rosenbrock method was used to perform the simulation in time domain. Rosenbrock methods are applicable for nonlinear systems. They are best suited for systems of stiff differential equations as explicit integration methods perform nonsatisfyingly for those problems.
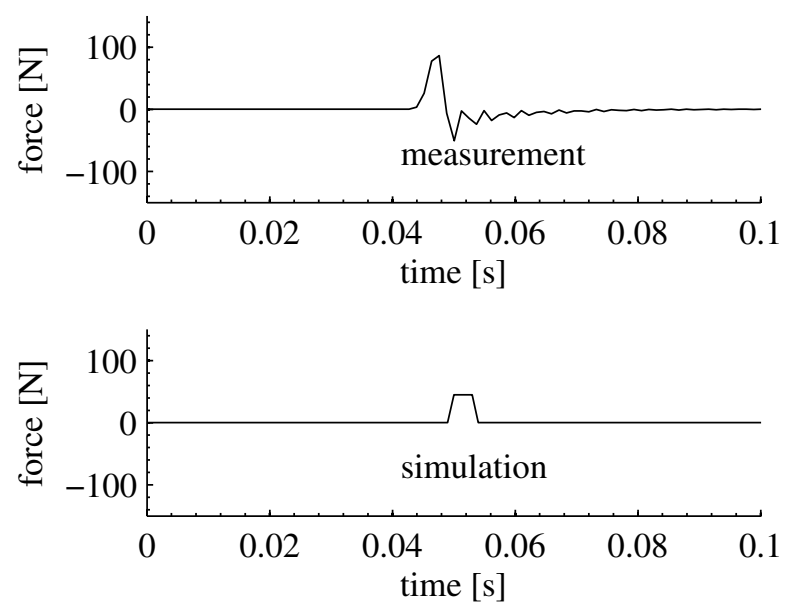

Fig. 10 Low impact force in measurement and simulation at a frequency of revolution of $125 \mathrm{~Hz}$, connection clamp stays closed.

The algorithms used for the simulation is a linear implicit 2nd and 3rd order Runge-Kutta method with step control.

After each time step the state of the clamp is checked with the condition of equation (4). If the state of the clamp turned out to be different from the state which was assumed for the calculation in the time step, this time step is repeated with the new state of the clamp.

\subsection{Results of the nonlinear simulation}

The simulation was performed in a first step at a frequency of revolution of $125 \mathrm{~Hz}$. The unbalance load was adjusted to the oscillation measured prior to the release of the connection. The mass of unbalance is $0.25 \mathrm{~g}$ and the radius of unbalance is $109.34 \mathrm{~mm}$.

The height and the duration of the impact acting on the motor base were adjusted to the measured impact (figure 10 and figure 12).

After the first impact in the test the motor base starts to oscillate. The impact hammer is not moved away fast enough. Hence it bounces against the motor base again. This explains the oscillations which could be observed in the measurement in figure 10 and figure 12 .

In figure 11 and figure 13 the computed and measured accelerations of the motor base are depicted. Simulation and measurement results coincide well. The simulation reflects also the phenomenon that increased amplitudes like in figure 13 occur only if the impact force is high enough (figure 12). If the impact is lower (figure 10), the clamp stays closed and the amplitudes diminish (figure 11). 

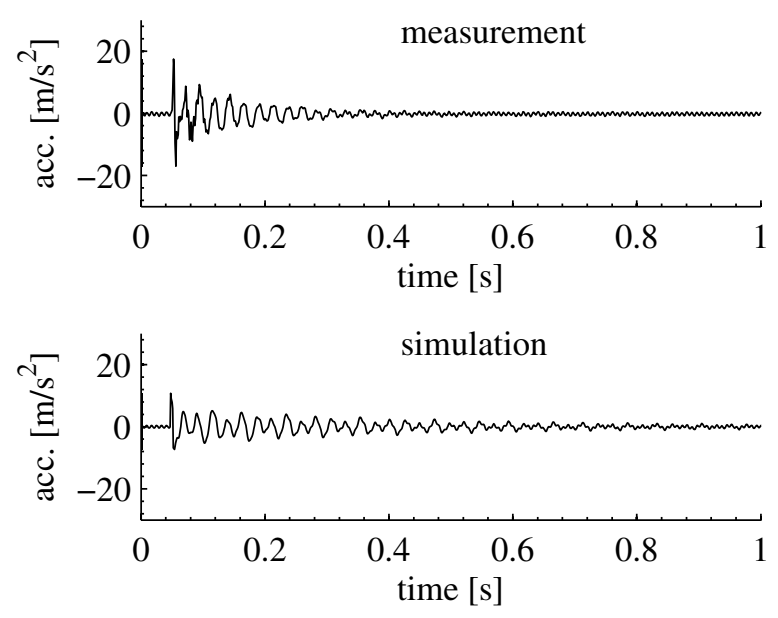

Fig. 11 Measured and computed acceleration of motor base at a frequency of revolution of $125 \mathrm{~Hz}$, low impact force.
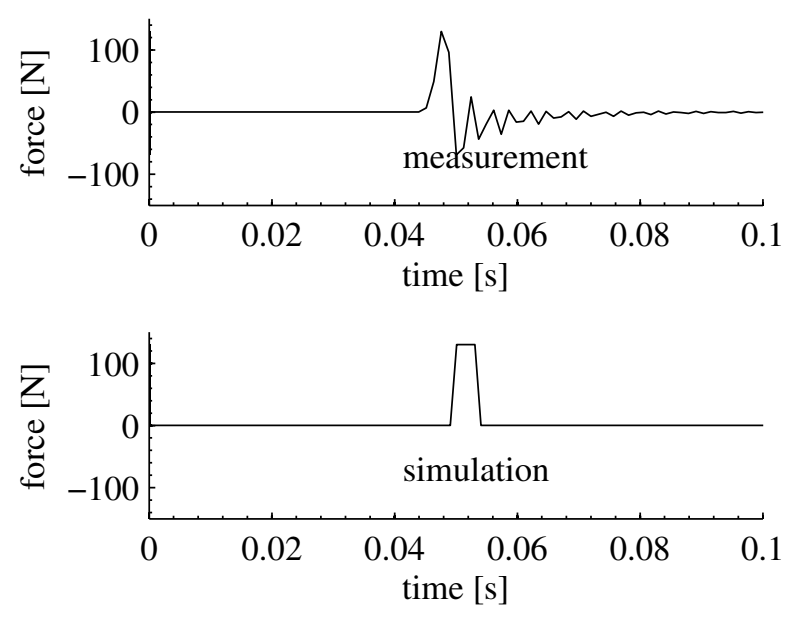

Fig. 12 High impact force in measurement and simulation at a frequency of revolution of $125 \mathrm{~Hz}$, connection clamp opens.
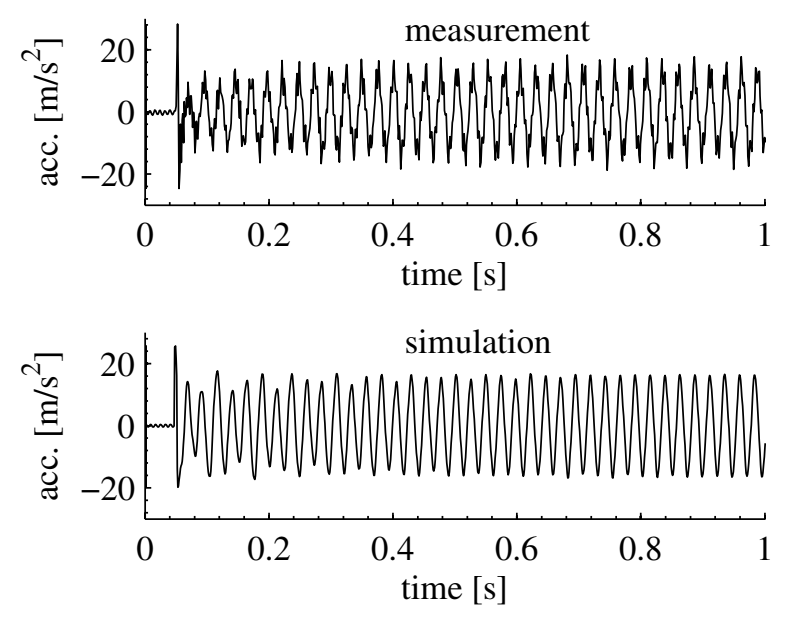

Fig. 13 Measured and computed acceleration of motor base at a frequency of revolution of $125 \mathrm{~Hz}$, high impact force.
The amplitude of the oscillation after the release of the clamp relies mostly on the parameters of o-ring and plastics, that means on damping, stiffness and clearance parameters.

The acceleration signals after the impact are analyzed via fast fourier transformation. The results are shown in figure 14 and 15.

If the connection clamp opens and bounded self-excited

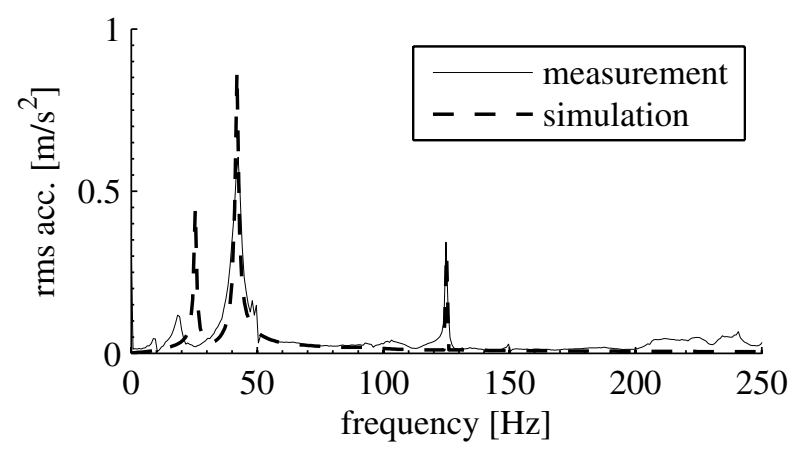

Fig. 14 RMS spectrum of time signal of figure 13 at a frequency of revolution of $125 \mathrm{~Hz}$, connection clamp open.

vibrations occur, the highest forward whirl below of the frequency of revolution of $125 \mathrm{~Hz}$ turned out to be the most dominant peak in the frequency spectrum (figure 14). This result holds for measurement and simulation. The highest forward whirl at this speed of revolution is $39.2 \mathrm{~Hz}$ (measurement) and $41.0 \mathrm{~Hz}$ (simulation).

It agrees with results from analytical research in internal damping. If the internal damping is of linear viscous damping character, it is known that the damping values of the forward whirls can get negative such that the frequency of the self-excited vibration is the same like the corresponding forward whirl (e.g. [14]).

In [15] a two degrees of freedom rotor with internal dry friction is analyzed analytically. It is denoted, that in the post critical speed range a bounded self-excited oscillation with the frequency of the first forward whirl occurs. In our case the same kind of dry friction is working in the clamp once the connection is released.

As the rotor shaft connection is stiffer, if the clamp is closed, the frequency value of the considered forward whirl is higher (figure 15, 42.4 Hz (measurement) and $42.0 \mathrm{~Hz}$ (simulation)). There is still positive damping such that the frequency is not dominating in the RMS spectrum in the same manner like for the open clamp. 


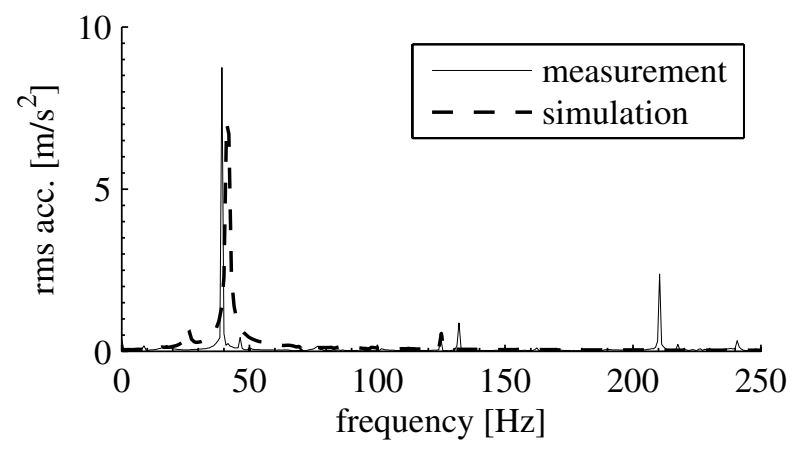

Fig. 15 RMS spectrum of time signal of figure 11 at a frequency of revolution of $125 \mathrm{~Hz}$, connection clamp closed.

Because of the nonlinearities in the force laws of o-ring and plastics the amplitudes for the case of an open clamp are bounded. Curve A in figure 16 shows the magnitudes of the motor base accelerations. If we neglect the relative stiffness $k_{i r}$ of the o-ring in equation (8) we obtain the force law of equation (7). Now only cubic nonlinearities are taken into account. This corresponds to curve B (figure 16). In curve $\mathrm{C}$ the cubic stiffness $k_{i n l}$ of o-ring and plastics is reduced to $10 \%$ of the original value and in curve D to $1 \%$ of the original value. It is obvious that the amplitudes of the motor base increase the smaller the nonlinearities of the joint stiffness are.

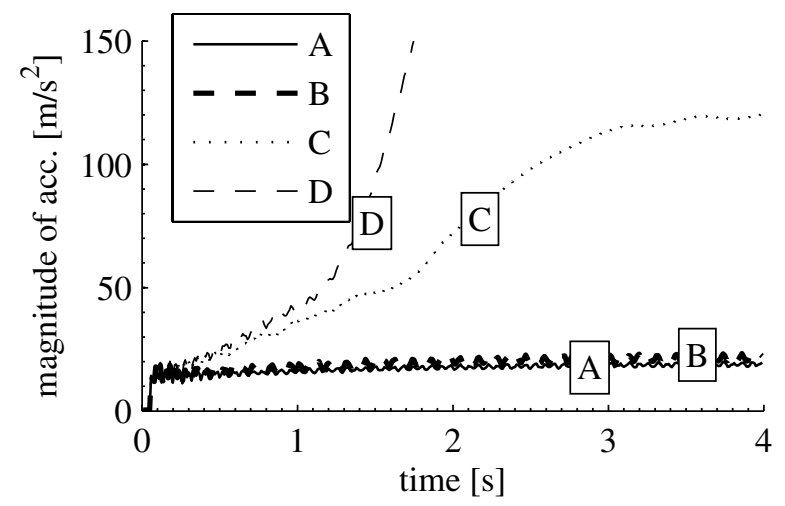

Fig. 16 Magnitude of motor base accelerations with varied joint parameters: A: original parameters like in figure 13, B-D: force law of equation (7) for o-ring and plastics, C: $10 \%$ cubic stiffness $k_{i n l}$ of B, D: $1 \%$ cubic stiffness $k_{i n l}$ of B.

In figure 17 measurement and simulation results at a frequency of revolution of $150 \mathrm{~Hz}$ are shown. The height of the impact force is $230 \mathrm{~N}$ in measurement and simulation.

A run-up and a run-out is depicted in figure 18. The same radius of unbalance like in figure 14 and 15 is used but the unbalance mass is augmented to $10 \mathrm{~g}$.

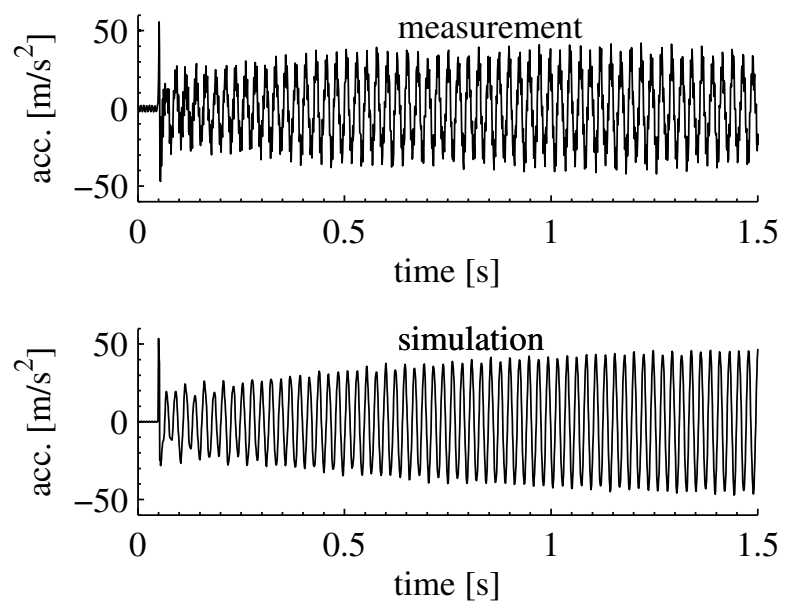

Fig. 17 Measured and computed acceleration of motor base, high impact force at a frequency of revolution of $150 \mathrm{~Hz}$.

The clamp opens at a frequency of revolution of 114 Hz. The clamp stays open, even if the system is shut down. The speed of revolution is changed linear from 0 to $210 \mathrm{~Hz}$ in 7 seconds.

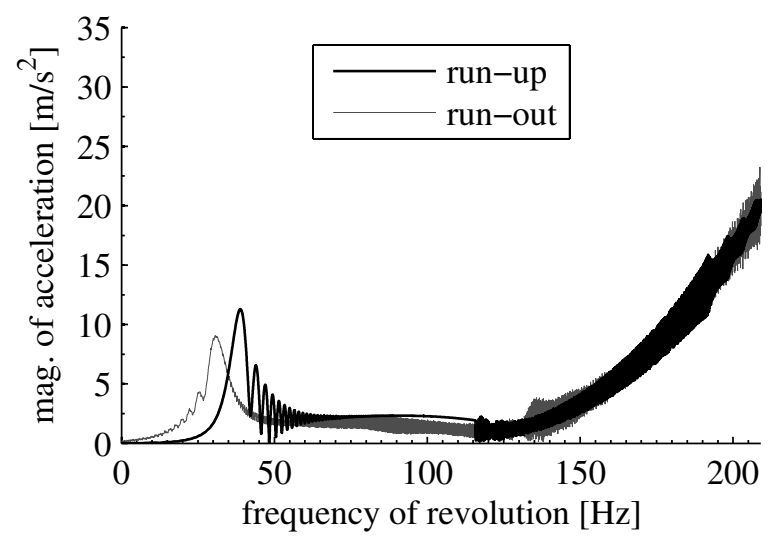

Fig. 18 Run-up and run-out of the lab centrifuge. Magnitude of accelerations at motor base.

\section{Conclusion}

The description of different parts of the rotor shaft joint of a lab centrifuge by different nonlinear force laws has proved to be a promising way in simulation. The nonlinear simulation results and the related measurements are in good agreement. It is possible to show the influence of internal damping in combination with nonlinear stiffness and dry friction to the behaviour of the device numerically. The nonlinearity of the rotor shaft connection turned out to be responsable for the limitation of 
the amplitudes once the internal damping of the connection starts to work in the post critical speed range. Measurement and simulation results coincide with results which are known from analytical models with internal damping.

\section{References}

1. Gasch, R., Nordmann, R. , Pfützner, H.: Rotordynamik. Springer Verlag, Berlin, Heidelberg, New York (2002)

2. Cowper: The shear coefficient in timoshenko's beam theory. ASME J. Appl. Mech. 33 (2), 335-340 (1966)

3. Shaw, J., Shaw, S.: Instabilities and bifurcations in a rotating shaft. J. Sound Vib. 132 (2), 227-244 (1989)

4. Genin, J., Maybee, J. S.: Stability in the three dimensional whirling problem. Int. J. Non. Lin. Mech. 4 (3), 205-215 (1969)

5. Tondl, A.: Some Problems of Rotor Dynamics. Chapman \& Hall, London (1965)

6. Dahl, P.: A solid friction model. Tech. rep., The Aerospace Corporation (May 1968)

7. Menq, C., Griffin, J., Bielak, J.: The influence of a variable normal load on the forced vibration of a frictionally damped structure. ASME J. Eng. Gas Turbines Power 108, 300-305 (1986)

8. Shoukry.: A mathematical model for the stiffness of fixed joints between machine parts. In: Proceedings of the NUMETA 85 Conference, pp. 851-8. Swansea, UK (1985)

9. Burdekin, M., Cowley, A., Back, N.: An elastic mechanism for the microsliding characteristics between contacting surfaces. J. Mech. Eng. Sci. 20 (3), 121-127 (1978)

10. Wettergren, H.: Material and microslip damping in a rotor taking gravity and anisotropic bearings into account. ASME J. Vib. Acoust. 131, 30-35 (2001)

11. Charleux, D., Gibert, C., Thouverez, F., Dupeux, J.: Numerical and experimental study of friction damping in blade attachments of rotating bladed disks. Int. J. Rotating Machinery 2006, 1-13 (2006)

12. Nacivet, S., Pierre, C., Thouverez, F., Jezequel, L.: A dynamic lagrangian frequency-time method for the vibration of dry-friction-damped systems. J. Sound Vib. 265, 201-219 (2003)

13. Beitz, W., Grote K.(Eds.).: Dubbel, Taschenbuch für den Maschinenbau. Springer (1997)

14. Fischer, J., Strackeljan,J.: Stability analysis of high speed lab centrifuges considering internal damping in rotor-shaft joints. Technische Mechanik 26 (2), 131-147 (2006)

15. Ishida, Y., Yamamoto, T.: Forced oscillations of a rotating shaft with nonlinear spring characteristics and internal damping. Nonlinear Dyn. 4, 413-431 (1993) 